\title{
Percepção materna sobre o comportamento de crianças com transtorno do espectro autista e a atuação de profissionais na intervenção multidisciplinar
}

\author{
Maternal perception of the behavior of children with \\ autism spectrum disorder and the performance of \\ professionals in the multidisciplinary intervention
}

LARISSA Lima DOS-SANTOS-SOUSA ${ }^{1}$ Luara laiane de Castro Moura ${ }^{\mathrm{I}}$ Danyel Pinheiro Castelo BRANCOII

ANDré PonTes-Silva ${ }^{\text {III }}$

${ }^{I}$ Faculdade Santo Agostinho, Teresina/PI - Brasil

"Universidade Metodista de São Paulo, São Paulo/SP - Brasil

IIIUniversidade Federal do Mara-

nhão, São Luís/MA - Brasil
Resumo - Introdução: o Transtorno do Espectro Autista (TEA) está relacionado a déficits nos comportamentos sociocomunicativos associados a movimentos estereotipados. Objetivo: descrever as dificuldades que as crianças com TEA apresentam nas atividades da vida diária, segundo as observações e descrições de suas mães. Metodologia: um estudo transversal com abordagem mista (quali-quantitativa). 40 mães do grupo de famílias assistidas cadastradas em uma associação de amigos do autista, na Região Nordeste do Brasil, foram recrutadas por meio de ligações. Resultados: a amostra possui idades de 25-67 anos, a maior parte dos diagnósticos investigados ocorreu entre o primeiro e o terceiro ano de vida, as alterações de sensibilidade periférica foram as mais observadas e o médico foi o profissional com maior quantitativo de participação. Conclusão: $90 \%$ das mães consideram importante a atuação multidisciplinar na intervenção do TEA em crianças de até 12 anos, e, entre as diversas manifestações relatadas, destacou-se que crianças com TEA expõem adversidades em se relacionarem e lidarem com situações que se diferem da rotina de atividades da vida diária.

Palavras-chave: Transtorno do Espectro Autista; Transtorno Global do Desenvolvimento; Tratamento Multidisciplinar.

Abstract - Purpose: To describe the difficulties that children with ASD present in activities of daily living, according to their mothers' observations and descriptions. Methods: cross-sectional study with a mixed approach (qualitative and quantitative). Through telephone calls, 40 mothers from the group of assisted families registered in an association of friends of the autistic person in the Northeast region of Brazil were recruited. Results: the sample is in the age group from 25 to 67 years old, most of the diagnoses investigated occurred between the first and the third year of life, changes in peripheral sensitivity were the most observed and the physician was the professional with the greatest participation. Conclusions: $90 \%$ of mothers consider the multidisciplinary role of intervention in ASD to be important in children up to 12 years of age and, among the various manifestations reported, it was highlighted that children with ASD have adversities in relating and dealing with situations other than the daily activities of life daily.

Keywords: Autism Spectrum Disorder; Global Developmental Delay; Multidisciplinary Treatment. 


\section{INTRODUÇÃO}

De acordo com o manual diagnóstico e estatístico de transtornos mentais DSM-V, ${ }^{1}$ o Transtorno do Espectro Autista (TEA) está relacionado a déficits na comunicação social e na reciprocidade. As especificidades dos comportamentos sociocomunicativos de pessoas com TEA constituem um quadro clínico que outrora foi chamado exclusivamente de autismo. ${ }^{2}$ Algumas pesquisas o descrevem como um transtorno invasivo no desenvolvimento que envolve dificuldades nas habilidades sociais e comunicativas, além daquelas atribuídas ao transtorno global do desenvolvimento, acompanhadas de comportamentos e interesses ora limitados, ora repetitivos, ora atípicos. É possível encontrar descrições afirmando que, nesses indivíduos, ocorre uma baixa modulação sensorial frente aos estímulos externos. ${ }^{3,4,5}$

Os padrões repetitivos e estereotipados, além dos desvios no componente sociocomunicativo, são característicos do TEA e incluem resistência a mudanças, insistência em determinadas rotinas, apego excessivo a objetos, fascínio com o movimento de peças e dificuldades de expressão verbal. Inclusive, às vezes observamos crianças com TEA entretidas por um longo período em determinadas brincadeiras, as quais, na verdade, a preocupação está centralizada em alinhar e/ ou organizar os brinquedos do que em usá-los para sua finalidade simbólica. É comum ver, também, estereotipias motoras e verbais, tais como se balançar e bater palmas repetitivamente, andar em círculos ou repetir determinadas palavras, frases e/ou canções. Ademais, o TEA é uma condição que apresenta ampla variabilidade de sintomas, ou seja, porque nem todas as pessoas com TEA possuem um comprometimento igual ao da outra. Haja vista que o TEA vai de um nível muito leve a um nível mais grave., Atualmente, existe uma grande preocupação atribuída ao estudo do TEA, em virtude do aumento considerável no número de casos reportados nos últimos anos, principalmente com ênfase na pediatria. ${ }^{8,9}$

Do ponto de vista epidemiológico a prevalência do autismo tem crescido progressivamente. Os Centros de Controle de Doenças e Prevenções estimam a prevalência de 1 em 68 crianças, sendo a relação masculino-feminino de 4, 5:1. ${ }^{9}$ Esse aumento pode estar ligado à evolução científica e tecnológica dos critérios diagnósticos, principalmente depois da quinta edição do Manual. ${ }^{1}$

O TEA está relacionado a alterações do Sistema Nervoso Central (SNC), isso é uma informação de comum acordo entre os especialistas, que provoca uma disfunção no desenvolvimento da criança. A neuropatologia do autismo possibilita a identificação do seu impacto sobre as características do cérebro que não podem ser avaliadas por meio de estudos de neuroimagem, como processos de diferenciação e migração, morfologia neuronal e distribuição espacial ou alterações cito arquitetônicas, as quais são, hoje, a neuropatogênese do TEA. Portanto, em consequência da complexidade do SNC, assim como da grande variabilidade de sintomas e apresentações fenotípicas artísticas, até o momento, não foi possível determinar qualquer aspecto biológico, ambiental, ou da interação de ambos, que pareça ser a etiologia certa para causar as alterações de sua patogênese. ${ }^{9}$

A incidência de diagnósticos do TEA tem crescido de forma significativa em vá- 
rios países, especialmente durante as últimas décadas, entretanto, esse avanço no número de diagnósticos não tem sido expressado positivamente no Brasil. ${ }^{6,9}$ Isso pode ser preocupante pelo fato de que a intervenção precoce depende da identificação e diagnóstico o mais breve possível - podem partir da análise de dificuldades específicas na orientação para estímulos sociais, contato ocular social, atenção compartilhada, imitação motora e jogo simbólico. 5,6,7,9,10

Algumas das principais metas do tratamento visam propiciar a aprendizagem cognitiva, social e da linguagem, reduzir a rigidez e as estereotipias, eliminar comportamentos contestáveis e diminuir o estresse da família. A eficácia do teste necessita da experiência e do conhecimento dos profissionais sobre o TEA e das suas habilidades para trabalhar em uma equipe multidisciplinar e com a família. ${ }^{11}$ Equipes multidisciplinares são formadas por vários profissionais, de diferentes áreas, que buscam abranger os diferentes aspectos de uma mesma situação.

$\mathrm{O}$ advento da tecnologia e os vários estudos sobre o TEA foram capazes de despertar certa preocupação a nível social, ${ }^{12}$ atualmente esses indivíduos recebem apoio e tratamento em uma organização não governamental chamada: Associação de Amigos do Autista (AMA), a qual consiste em uma instituição filantrópica que atende crianças, jovens e adultos com TEA e outras síndromes, e conta com uma equipe terapêutica composta por: Psicólogos, Assistentes Sociais, Fonoaudiólogos, Professores, Fisioterapeutas, Profissionais de Educação Física e demais profissionais, auxiliando no processo avaliativo e clínico dos pacientes.

A AMA serve como espaço de apoio ao cuidar do filho desde sua criação e representa um local onde as mães e as crianças com TEA depositam parte de suas possibilidades. Muito tem contribuído para que essas crianças possam desenvolver a sociabilização, a comunicação, a imaginação e, até mesmo, realizar pequenas tarefas como a de pegar em um lápis para escrever o próprio nome. ${ }^{13}$ Embora o TEA não seja um transtorno de descoberta recente, no Estado do Piauí a chegada da AMA e dos profissionais envolvidos têm difundido pela mídia esse assunto e, de alguma forma, despertado interesse na sociedade civil para melhor conhecer/ajudar as pessoas com TEA e seus familiares. ${ }^{12,13}$

Diante dessa experiência surgem alguns questionamentos, a saber: qual é a percepção materna a respeito da atuação multidisciplinar na intervenção do TEA? O que as mães de crianças com diagnóstico de TEA observam sobre as dificuldades que os filhos apresentam nas atividades da vida diária? Nessa perspectiva, o presente estudo teve como objetivo descrever as dificuldades que crianças com TEA apresentam nas atividades da vida diária, segundo as observações e descrições de suas mães.

\section{Metodologia}

Um estudo transversal com abordagem qualitativa. Elaborou-se um formulário, contendo 6 domínios, o qual, por sua vez, foi preenchido com dados de 40 mães piauienses com faixas etárias entre 25 e 67 anos, responsáveis por 40 crianças com TEA diagnosticadas e inscritos na Associação de Amigos do Autista em Teresina (PI).

Os critérios de inclusão incluíram: ser mulher, com mais de 18 anos, e ser mãe 
de uma criança com diagnóstico do TEA. As mães recrutadas para este artigo foram distinguidas levando em consideração a frequência e o maior número de consultas e retornos entre janeiro de 2015 e maio de 2016. Por meio de amostragem intencional, a proposta de pesquisa foi apresentada por meio de chamada para telefone/celular, dessa forma, as mães foram convidadas a participar e as 40 mulheres concordaram. O preenchimento dos formulários - via sistema de controle da AMA - ocorreu em abril de 2016. A pesquisa foi aprovada pelo comitê de ética em pesquisa com seres humanos (número do parecer 1397575).

Após o preenchimento dos formulários e da chamada para os números de telefone/ celular disponíveis, confirmou-se o agendamento (data e hora) para cada mãe. Em todas as entrevistas, os pesquisadores procuraram preparar o local, para oferecerem um ambiente calmo e adequado o suficiente para realizarem genuínos encontros fenomenológicos. Cada entrevistada concentrou-se em responder duas perguntas: I) Você considera importante a atuação multidisciplinar da AMA para o tratamento do TEA?; II) Quais dificuldades você percebe nas atividades da vida diária do/a seu/sua filho/a? Todas as participantes assinaram o termo de consentimento livre e esclarecido. Os dados foram apresentados em percentual (\%).

\section{RESUlTAdos E Discussão}

De acordo com a análise dos dados coletados, $22,5 \%$ das mães correspondem à faixa etária de 31 a 48 anos e $42,5 \%$ das crianças foram diagnosticadas com idades entre 3 e 6 anos (Tabela 1). Em relação à idade das mães, esse estudo corrobora com os achados da literatura, todavia, a faixa etária das crianças diagnosticadas apresenta divergência, evidenciando um diagnóstico tardio. ${ }^{5,14}$

As alterações mais observadas na criança com TEA, nesta pesquisa, referem-se a alterações posturais $(52,5 \%)$ e de sensibilidade periférica (60\%) (Tabela 1). Esses achados concordam com evidências que descrevem que a criança com TEA apresenta dificuldades de compreender seu corpo em sua globalidade e em segmentos, assim como seu corpo em movimento, uma vez que, distúrbios na estruturação do esquema corporal, hipoestesia e debilidade proprioceptiva prejudicam também o desenvolvimento do equilíbrio estático, postural, lateralidade e noção de reversibilidade, que são funções de base necessárias à aquisição da autonomia e aprendizagens cognitivas. ${ }^{6,15}$

Acerca dos profissionais inclusos no tratamento (Tabela 1), a maior parte foram médicos (57,2\%), talvez isso seja explicado pela necessidade do diagnóstico nas primeiras consultas, todavia, vale ressaltar que, na medicina - em sua atuação isolada, isto é, sem atuação multidisciplinar - os profissionais não estão preparados, de forma literal, para detectar o TEA, isso gera riscos e pode impossibilitar uma intervenção precoce em crianças com TEA. ${ }^{16,8-10}$ Sobre a percepção materna no que diz respeito à atuação multidisciplinar para o tratamento do TEA, $90 \%$ das mães consideram o atendimento multidisciplinar importante para o tratamento, e $10 \%$ das mães manifestaram indiferença sobre o assunto. 
Tabela 1 - Dados sociodemográficos de 40 mães cadastradas no sistema da Associação de Amigos do Autista em Teresina (PI), durante o mês de abril, 2016.

\begin{tabular}{|c|c|}
\hline Variáveis & $\%$ \\
\hline \multicolumn{2}{|l|}{ Idade das mães entrevistadas } \\
\hline $25-30$ anos & 15,0 \\
\hline $31-36$ anos & 22,5 \\
\hline $37-42$ anos & 22,5 \\
\hline $43-48$ anos & 22,5 \\
\hline 49-54 anos & 15,0 \\
\hline $55-60$ anos & 0,0 \\
\hline $61-67$ anos & 2,5 \\
\hline \multicolumn{2}{|l|}{ Idade de diagnóstico do TEA em seus filhos } \\
\hline $0-1$ ano & 7,5 \\
\hline $1-3$ anos & 45,0 \\
\hline 3-6 anos & 42,5 \\
\hline $9-12$ anos & 5,0 \\
\hline \multicolumn{2}{|l|}{ Alterações observadas na criança com TEA } \\
\hline Alteração postural & 52,5 \\
\hline Deficiência no equilíbrio & 32,5 \\
\hline Deficiência na coordenação & 50,0 \\
\hline Fraqueza muscular & 42,5 \\
\hline Alteração de sensibilidade periférica & 60,0 \\
\hline \multicolumn{2}{|l|}{ Profissionais inclusos no tratamento } \\
\hline Médico & 57,2 \\
\hline Psicólogo & 9,8 \\
\hline Fonoaudiólogo & 9,8 \\
\hline Fisioterapeuta & 3,2 \\
\hline Terapeuta & 3,8 \\
\hline Nutricionista & 2,8 \\
\hline Profissional de Educação Física & 1,2 \\
\hline Pedagogo & 9,5 \\
\hline Outros & 2,7 \\
\hline
\end{tabular}

Considera importante a atuação multidisciplinar para o tratamento do TEA?

\begin{tabular}{c|c}
\hline Sim & 90,0 \\
\hline Não & 10,0
\end{tabular}

Fonte: elaborado pelos autores (2020). 
Mediante os dados obtidos nesta pesquisa foi possível verificar que os sintomas mais notáveis do TEA são demonstrados após os três primeiros anos de vida da criança, tendo como principais características: dificuldades de se relacionar com pessoas, atraso na fala e insistência obsessiva em manter a monotonia da rotina, informações apresentadas também na literatura. ${ }^{11,14,17}$ As alterações de equilíbrio, coordenação motora e fraqueza muscular, mesmo apresentando um percentual menor, quando comparadas às alterações posturais e de sensibilidade (Tabela 1), merecem atenção desde cedo, haja vista que as atividades musculares e exercícios de coordenação motora devem ser realizadas desde o nascimento, pois o treinamento desses componentes relaciona-se com a manutenção das capacidades físicas. ${ }^{18,19,20}$

Algumas mães descreveram que seus filhos com diagnóstico de TEA apresentam dificuldades em se relacionarem e lidarem com situações diferentes das de costume na rotina de atividades da vida diária, essas características, por sua vez, estão de acordo com as informações certificadas na literatura científica. ${ }^{17}$

Embora registrar a relevância da intervenção multidisciplinar no tratamento do TEA esteja sendo feita, existem pessoas que não conseguem ver essa dimensão, seja por falta de informação, seja por falta de conhecimento e compreensão, seja por outros motivos intrapessoais, desse modo, fica evidenciado que a propagação do mérito que o atendimento multidisciplinar tem direito se faz necessário. Nesta pesquisa, os sintomas mais notáveis são vistos e/ou diagnosticados após os três primeiros anos de vida da criança, caracterizando um problema relacionado ao diagnóstico tardio. Por fim, chama-se a atenção para a adequação dos profissionais envolvidos na intervenção do TEA, haja vista que a distribuição de atuações se concentrou na área da medicina (57,2\%), entretanto, deve-se destacar as importâncias e especificidades que cada uma das profissões envolvidas possui, uma vez que, no século XXI, predomina-se a abordagem biopsicossocial em detrimento do modelo biomédico. ${ }^{16,8-10}$

\section{CONSIDERAÇõeS FINAIS}

A maior parte da amostra (90\%) considera importante a atuação multidisciplinar na intervenção do TEA em crianças de até 12 anos. De acordo com as entrevistas, crianças com TEA apresentam adversidades em se relacionarem e lidarem com situações que se diferem da rotina de atividades da vida diária.

\section{REFERÊNCIAS}

1. American Psychiatric Association. DSM-5: Manual diagnóstico e estatístico de transtornos mentais. São Paulo: Artmed Editora, 2014.

2. Carvalho FSS Filha, Nascimento IBR, Santos JC, SIlva MVR, Moraes IM Filho, Viana LMM. Análise do comportamento aplicada ao transtorno do espectro autista: aspectos terapêuticos e instrumentos utilizados-uma revisão integrativa. REVISA. 2019; 8(4):525-36

3. Octaviano AJM, Evaristo ALA, Carvalho BM, Fantacini RAF. A inclusão do aluno com transtorno do espectro autista na educação infantil. Res., Soc. Dev. 2019; 8(1):1-15 
4. Silva CCB, Ferreira AF, Portugal G. Inclusão escolar de uma criança com transtorno do espectro do autismo na educação infantil-contributos do sistema de acompanhamento das crianças. Indagatio Didactica. 2019; 11(4): 25-40

5. Bezerra OV, Silva MC, Lôbo AMR, Cidrão SC, Silva BCR, Santos SQ, et al. A Psicomotricidade Como Ferramenta Inclusiva da Criança Autista na Educação Infantil. BJD. 2020; 6(8): 54631-40

6. Heinze JS, Cruz RB, Volpasso MC, Souza VM, Ramos JRS. A cultura corporal de movimento e práticas equoterápicas como forma de autonomia e inclusão social de alunos-praticantes com transtorno do espectro autista. BJD. 2020; 6(8): 59375-80

7. Barcelos KS, Martins MFA, Betone GAB, Ferruzzi EH. Contribuições da análise do comportamento aplicada para indivíduos com transtorno do espectro do autismo: uma revisão. BJD. 2020; 6(6): 37276-91

8. Reis ST, Lenza N. A Importância de um diagnóstico precoce do autismo para um tratamento mais eficaz: uma revisão da literatura. Revista Atenas Higeia. 2020; 2(1): 1-7

9. Vieira AC. Autismo: As características e a importância do diagnóstico precoce [trabalho de conclusão de curso]. Manhuaçu: Centro Universitário UNIFACIG; 2019. 17 p.

10. Belo RA, Fonseca TC. A relação entre autismo, família e aprendizagem, em artigos da base de dados Scielo (2003-2019). Temas em Educ. e Saúde. 2020; 16 (1): 118-32

11. Oliveira RLG, Carvalho ACG. A percepção do enfermeiro no atendimento ao paciente autista. REINPEC. 2019; 5 (5): 228-235

12. Vidal NLC, Moreira PC. A importância da relação família, escola e psicólogo na escolha do tratamento do aluno com transtorno do espectro do autismo-TEA. In: Anais do 1st CINTEDI; 2014. Campina Grande: Realize Editora. p. 1-2

13. Carvalho-Filha FSS, Costa e Silva HM, Castro RP, Moraes-Filho IM, Nascimento FLS. Coping e estresse familiar e enfrentamento na perspectiva do transtorno do espectro do autismo. REVISA. 2019; 33(1):1-8

14. Rendon DCS, Salimena AMO, Amorim TV. Convivência com filhos com transtorno do espectro autista: desvelando sentidos do ser-aí-mãe.Rev. baiana enferm. 2019. 33 (1): 1-8

15. Dias HLA, Borragine SDO. A Inclusão de Crianças Autistas nas Aulas De Educação Física Escolar. REDE. 2020; 3(1): 1-12

16. Flores MR, Smeha LN. Bebês com risco de autismo: o não-olhar do médico. Ágora (Rio J.). 2013; $16(1): 141-57$

17. Sanini C, Ferreira GD, Souza TS, Bosa CA. Comportamentos indicativos de apego em crianças com autismo. Psicol. Reflex. Crit. 2008; 21(1):60-5

18. Silva AP, Silva RCA, Silva VLF. Os principais aspectos do Transtorno do Desenvolvimento da Coordenação nas atividades da vida diária de uma criança em fase de crescimento: um consenso científico. Saúde, Batatais. 2017; 6(1): 37-45

19. Smits-Engelsman B, Vinçon S, Blank R, Quadrado VH, Polatajko H, Wilson PH. Evaluating the evidence for motor-based interventions in developmental coordination disorder: A systematic review and meta-analysis. Res Dev Disabil. 2018; 74:72-102

20. Blank R, Barnett AL, Cairney J, et al. International clinical practice recommendations on the definition, diagnosis, assessment, intervention, and psychosocial aspects of developmental coordination disorder. Dev Med Child Neurol. 2019; 61(3):242-85

21. Poletti M, Tortorella A, Raballo A. Impaired Corollary Discharge in Psychosis and At-Risk States: Integrating Neurodevelopmental, Phenomenological, and Clinical Perspectives. Biol Psychiatry Cogn Neurosci Neuroimaging. 2019; 4(9):832-41 


\section{DADOS DOS AUTORES}

\section{LARISSA LIMA DOS-SANTOS-SoUsa}

Fisioterapeuta graduada pela Faculdade Santo Agostinho, Teresina/PI - Brasil.larissinha_lima_2006@ hotmail.com

\section{Luara laiane de Castro Moura}

Fisioterapeuta graduada pela Faculdade Santo Agostinho, Teresina/PI - Brasil. luara-layane@hotmail.com

\section{Danyel Pinheiro Castelo Branco}

Programa de Pós-graduação em Psicologia da Saúde pela Universidade Metodista de São Paulo, São Paulo/SP - Brasil. delcbs@hotmail.com

\section{André Pontes-Silva}

Programa de Pós-graduação em Saúde do Adulto (Mestrado Acadêmico da Área de Medicina), Universidade Federal do Maranhão, São Luís/MA - contato.andrepsilva@gmail.com

Submetido em: 2-5-2020

Aceito em: 4-5-2021 